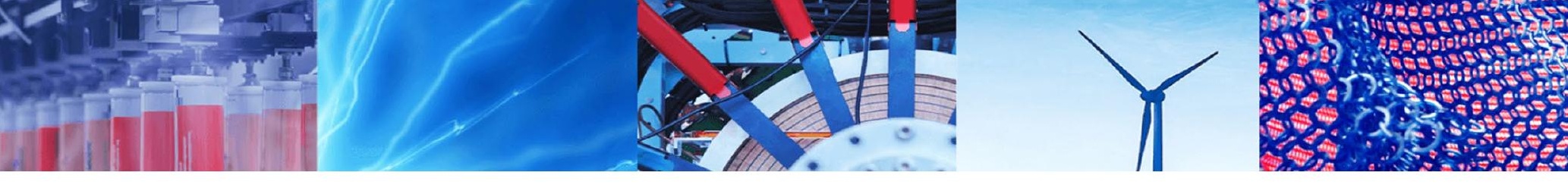

Research Article

\title{
Investigation of the effects of different parameters on the generated pressure waves inside the tunnels
}

\author{
Tahereh Izadi ${ }^{1,2} \cdot$ Omid Abouali $^{1} \cdot$ Mozaffar Ali Mehrabian $^{2} \cdot$ Mazyar Salmanzadeh $^{2}$
}

Received: 19 December 2019 / Accepted: 19 March 2020 / Published online: 22 July 2020

(c) Springer Nature Switzerland AG 2020

\begin{abstract}
In this research the train movement along the tunnel was simulated numerically, and the time evolution of the flow field in the tunnel was evaluated. The unsteady forms of continuity, Navier-Stokes, and energy equations were applied to solve the compressible viscous flow using the dynamic mesh technique. The importance of using a compressible flow model, even for the low Mach number flow case, was demonstrated. It was shown that even in low train speeds; a compressible model is preferred, as it can predict the pressure fluctuations, while an incompressible model may only be used for predicting the mean pressure. However, in cases where pressure fluctuations are not important, the incompressible model can be used especially for a train with low acceleration. When the trains accelerate, it was found that the initial slope of the pressure curve is proportional to the train acceleration. Therefore, the increase in the train acceleration increases the maximum pressure rise. Also, the first pressure rise depends on the velocity of the train tail as it enters the tunnel. In addition, the earlier equations in the literature for computing the maximum pressure rise, can properly predict the pressure rise of accelerating trains if the train tail velocity, as it enters the tunnel, is used as the velocity scale.
\end{abstract}

Keywords Pressure wave $\cdot$ Maximum pressure rise $\cdot$ Compressibility $\cdot$ Numerical simulation $\cdot$ Train acceleration

\section{Introduction}

The movement of high-speed trains causes significant changes in the airflow around the train as well as at a certain distance from the train. Obviously, the pressure and velocity change around the moving vehicle. This is more effective in closed spaces like tunnels. The pressure in front of the moving train increases; therefore, when the train head reaches the tunnel from outside ambient, a compression wave is generated and propagates along the tunnel at about the speed of sound. The arrival of the compression wave at each point, makes the pressure rise at that point: this is, however, the first and most considerable pressure rise occurring at that point. Besides, the pressure behind the train decreases; so, when the tail of the train enters the tunnel, an expansion wave is generated and propagates along the tunnel. These main waves reflect at the tunnel end with opposite sense. Thus, the pressure distribution inside the tunnel is influenced by the movement of theses pressure waves. The waves may make an aural discomfort for train passengers, and affect the tunnel ventilation; it is therefore important to investigate the effect of different parameters on pressure rise. The parameters which are more important are train velocity, train and tunnel sizes, train nose shape, atmospheric pressure, traffic condition, etc.

Ogawa and Fujii [1] numerically investigated the compression wave created by a high speed train entering a tunnel. They explained that the high pressure region generated at the train nose, propagates in the tunnel and becomes a one-dimensional compression wave. By studying the aerodynamic forces applied on the train, they

Tahereh Izadi, th.izadi@gmail.com | ${ }^{1}$ School of Mechanical Engineering, Shiraz University, Shiraz, Iran. ${ }^{2}$ Department of Mechanical Engineering, Shahid Bahonar University of Kerman, Kerman, Iran. 
concluded that the drag coefficient increases when the pressure increases in front of the train. The total increase of $C_{D}$ is 0.4 .

Researchers like Howe and Cox [2], Baron et al. [3] and Vardy [4] worked on micro-pressure waves. In general, when the propagating pressure waves reflect at an open end, part of their energy is released to the outside. These radiated waves are known as micro-pressure waves (MPWs). The magnitude of the micro-pressure waves is proportional to the maximum rate of pressure change at the tunnel exit. Nakao et al. [5] showed that when the first pressure rise is sufficiently high, the compression wave will convert to a shock wave. Takayama et al. [6] proved that this shock wave causes an unpleasant sonic boom to be emitted from the tunnel exit. They also examined different techniques, such as installation of entrance hoods, use of a porous or perforated wall, and a combination of them, to reduce the tunnel sonic boom. lida et al. [7] also worked on damping instruments like the entrance hoods.

Chen et al. [8], experimentally, showed that the piston effect and the airflow velocity in a tunnel are increased by increasing the vehicle speed and the ratio of vehicle crosssectional area to the tunnel cross-sectional area. This ratio is called the blockage ratio $\left(\beta=A_{\text {train }} / A_{\text {tunnel }}\right)$.

By different theoretical approaches, Pope and Woods [9], Howe [10] and Matsuo et al. [11] presented various equations for calculating the first pressure rise generated inside the tunnel. Most of these equations are based on two parameters: train velocity and blockage ratio. A shortcoming of these equations for estimating the maximum pressure rise for accelerating trains is that the appropriate train velocity needed to be used is not known and this is the issue which will be investigated in the present study. In addition, these equations only provide the magnitude of the first pressure rise for the entire length of the tunnel, but it was shown by Nakao et al. [5] and Takayama et al. [6] that the pressure waves propagating along the tunnel lose their intensity so that the maximum pressure rise gradually decreases.

Kim and Kim [12] investigated the unsteady threedimensional flow in a subway tunnel induced by the passage of train through the tunnel, experimentally and numerically. They used a $1 / 20$ scale model tunnel for their experimental research and applied the sharp interface method of CFX4 (a commercial CFD software) for their computational model.

For simulating the train-induced airflow inside the tunnel, two techniques can be applied in Fluent software package: sliding mesh technique, and dynamic mesh technique. In sliding mesh technique, two separate zones are built and connected to each other with grid interfaces. The moving zone slides over stationary zone at a defined velocity. Yang et al. [13], Khayrullina et al. [14] and Chen et al. [15] applied the sliding mesh technique, while Huang et al. [16], Liu et al. [17], Zhang et al. [18] and Izadi et al. [19] applied the dynamic mesh technique. In this technique, the shape of the domain is changing with time due to the domain moving boundaries. The dynamic mesh technique is suitable for investigating the propagation and reflection of the pressure waves inside the tunnels. Because, in sliding mesh technique, the geometry changes during train movement, and temporary wall surfaces are created at the boundaries where two interfaces do not overlap.

Bazdidi et al. [20] simulated a turbulent synthetic jet by two different technics for simulation of the actuator, namely, the mass flow inlet and the dynamic mesh method. They concluded that the compressible flow model presents more accurate results than the incompressible one.

Chu et al. [21] studied the generated pressure waves inside the tunnel when two trains passing each other, by sliding mesh technique. Their numerical results showed that the extremum pressure coefficients occur at the tunnel mid-point when two trains move towards each other with the same speed. Xue et al. [22] investigated the three-dimensional unsteady air flow in a subway station and tunnel numerically and experimentally. They concluded that the RNG $k-\varepsilon$ and standard $k-\varepsilon$ equations can be applied to simulate the airflow in the tunnel. Camelli et al. [23] showed that the LES model can well predict the train movement in a tunnel, but involving a high computational cost. Some researchers such as Wei et al. [24] and Jian-jun [25] investigated the effect of a PSD (platform screen door) on pressure variations inside the tunnel and Zhang et al. [26] analyzed the airflow rate through the entrances and adjustable platform doors (APDs) of subway systems. APD systems are installed in subway stations to reduce the energy consumption by utilizing train-induced airflow. Zhou et al. [27] investigated the natural ventilation in different sloping tunnels and proposed an iterative model to predict the velocity of airflow in city tunnels. Niu et al. $[28,29]$ simulated two adjacent platforms with a connecting tunnel using their numerical model. They studied the effects of some parameters such as the platform spacing, on the transient pressure inside the tunnel. Niu et al. [30] also analyzed the aerodynamic performance for different lengths of the tapered nose of the train. One of the main topics regarding the numerical investigation of piston effect inside the tunnel is the compressibility effect. In so researches of train-induced air flow such as [12,31-34], the flow is considered to be incompressible. While in some other researches, even with the same range of train velocity, $[15,24,28,35,36]$ the flow is assumed to be compressible. It is good to note that when high-speed trains move at a velocity of around 
$300 \mathrm{~km} / \mathrm{h}$, the compressible flow was necessarily applied $[13,20,29,37]$. On the other hand, a computer program named subway environment simulation (SES) was widely used for simulating the air flow inside the tunnels. However, the SES program is also limited, because it is based on one dimensional incompressible flow. Therefore, it is essential to investigate the effect of compressibility for the train-induced airflow and to determine the potential and benefits of compressible and incompressible models. Thus, in this research, the train-induced airflow and the piston effect inside a tunnel are investigated numerically. The dynamic mesh technique is employed to simulate the train movements in the outside ambient and along the tunnel. The effects of different parameters, including the flow compressibility, the train acceleration, and length, on the generated pressure waves and entry pressure rise are studied.

\section{Model description}

\subsection{Computational model}

The flow field around a moving train and inside the tunnel at different time steps is obtained using the computational model. To achieve this aim, a cube is assumed to represent the train. An appropriate structured computational grid is generated. The computational domain and the corresponding computational grid are shown in Fig. 1.

The height of the first cells near the walls, $y$, should be selected in a way that the non-dimensional $\mathrm{y}^{+}$value is maintained in an appropriate range which is $30<\mathrm{y}^{+}<300$, where $y^{+}=\frac{\rho u_{\tau} y}{\mu}$. After analyzing the grid size sensitivity, around 2 million cells for the 3-D model and around 50,000 cells for the alternative axisymmetric model are employed. The train is stopped in the outside ambient, near the tunnel entrance $(t=0 \mathrm{~s})$. As shown in Fig. 1, to separate the moving grids from the stationary ones, a grid interface, thoroughly surrounding the train along the tunnel and outside ambient, is used.

When the train moves, the computational mesh changes, it is compressed or stretched in the region around the train. So it must be updated at each time step. To regenerate the computational mesh, the layering method of the dynamic mesh technique of FLUENT software package is used.

In this method, a starting computational grid is generated. Two surfaces inside the grid interface, at the front and the back of the train are defined as moving boundaries. The description of the train motion is imported

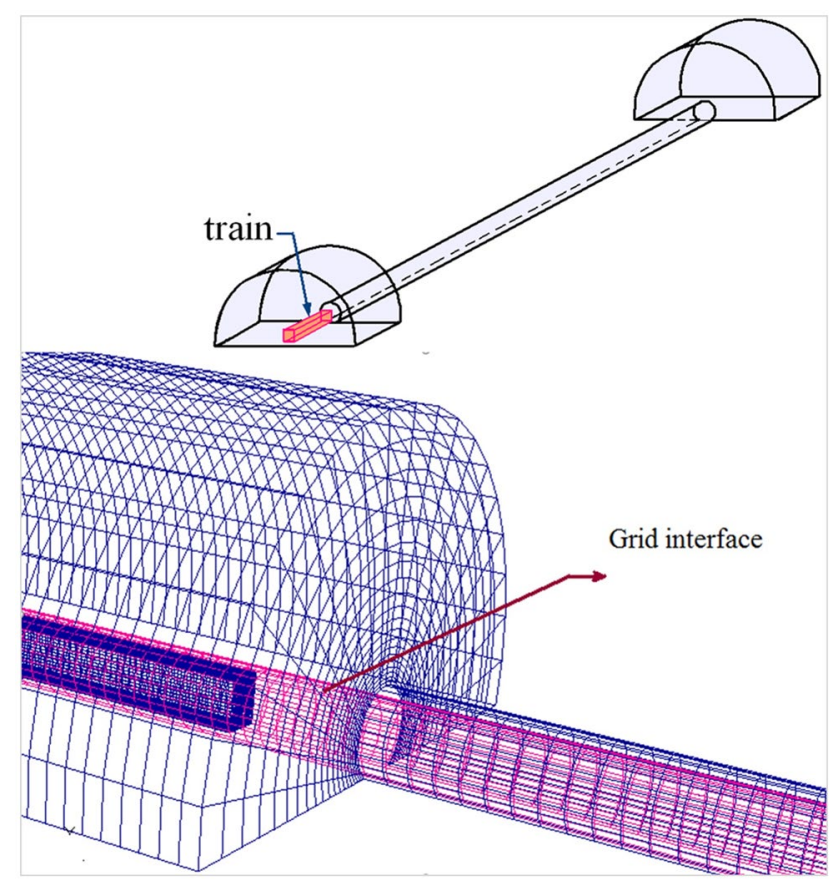

Fig. 1 Schematic diagram of computational domain at the initial time with the grid interface surrounding the train along the tunnel and outside ambient

using user defined functions (UDFs). The layer of cells next to a moving boundary is removed or added based on the cells height.

The cells adjacent to the front of a moving train are being compressed, as far as the following condition is met:

$h_{\min }<\alpha_{c} h_{\text {ideal }}$

where $h_{\text {ideal }}$ is the initial width of the cell. When this condition is met, the compressed layer of cells is merged into one layer.

Behind the train, the cells adjacent to a moving train are expanding as far as the following condition is met:

$h_{\min }>\left(1+\alpha_{s}\right) h_{\text {ideal }}$

When this condition is met, the cells in adjacent layer will split into two layers. $\alpha_{s}$ and $\alpha_{c}$ are the factors which determine the extent of expanding and compressing.

\subsection{Time step definition}

To select the appropriate time step, two parameters should be considered: (1) The time spent by the pressure waves to travel along the tunnel with the speed of sound, a, and (2) the length of the cell divided by the train speed. To capture the effect of wave propagations on pressure distribution at any point along the tunnel, the time step should be less 
than $\mathrm{L}_{\text {tunnel }} / \mathrm{a}$; thus, $\Delta t_{1}=\frac{L_{\text {tunnel }}}{a}$. On the other hand, based on dynamic mesh restriction, the train movement at each time step, should be less than the size of layer next to the moving boundary; thus, $\Delta t_{2}=\frac{L_{\text {cell }}}{V}$. The smaller time step between $\Delta t_{1}$ and $\Delta t_{2}$ is selected for the unsteady solution of this moving mesh problem. Thus, for train velocity shown in Fig. 7a, a characteristic time step of $\Delta t=0.01 \mathrm{~s}$ is applied for this research.

\section{Numerical method}

The continuity and reynolds averaged Navier-Stokes (RANS) equations are used to simulate the unsteady train-induced viscous flow inside the tunnel. Both compressible and incompressible flow models are studied and compared. Since the flow is turbulent, the standard k- $\varepsilon$ model is also employed.

The continuity, the Reynolds-averaged Navier-Stokes equations and the transport equation of the total energy, E can be written as:

$\frac{\partial \rho}{\partial t}+\frac{\partial}{\partial x_{i}}\left(\rho u_{i}\right)=0$

$$
\begin{aligned}
& \frac{\partial\left(\rho u_{i}\right)}{\partial t}+\frac{\partial}{\partial x_{j}}\left(\rho u_{i} u_{j}\right)=-\frac{\partial P}{\partial x_{i}}+\rho g \delta_{i 3} \\
& +\frac{\partial}{\partial x_{j}}\left[\mu\left(\frac{\partial u_{i}}{\partial x_{j}}+\frac{\partial u_{j}}{\partial x_{i}}-\frac{2}{3} \delta_{i j} \frac{\partial u_{l}}{\partial x_{l}}\right)\right] \\
& +\frac{\partial}{\partial x_{j}}\left(-\rho \overline{u_{i}^{\prime} u_{j}^{\prime}}\right)
\end{aligned}
$$

$$
\begin{aligned}
& \frac{\partial}{\partial t}(\rho E)+\frac{\partial}{\partial x_{i}}\left[\mu_{i}(\rho E+P)\right] \\
& =\frac{\partial}{\partial x_{j}}\left(k_{e f f} \frac{\partial T}{\partial x_{j}}+\mu_{i}\left(\tau_{i j}\right)_{\text {eff }}\right) \\
& \left(\tau_{i j}\right)_{e f f}=\mu_{\text {eff }}\left(\frac{\partial u_{j}}{\partial x_{i}}+\frac{\partial u_{i}}{\partial x_{j}}\right)-\frac{2}{3} \delta_{i j} \mu_{e f f} \frac{\partial u_{k}}{\partial x_{k}}
\end{aligned}
$$

where $u_{i}, P, \mathrm{~T}$ and $\rho$ are the mean velocity, pressure, temperature, and air density, respectively. $\mu$ is the air molecular viscosity, $k_{\text {eff }}$ is the effective thermal conductivity and $\left(\tau_{i j}\right)_{\text {eff }}$ is the deviatoric stress tensor which is defined by Eq. (4). By the Boussinseq approximation, the stress tensors are related to the mean velocity gradients:

$-\rho \overline{u_{i}^{\prime} u_{j}^{\prime}}=\mu_{t}\left(\frac{\partial u_{i}}{\partial x_{j}}+\frac{\partial u_{j}}{\partial x_{i}}\right)-\frac{2}{3}\left(\rho k+\mu_{t} \frac{\partial u_{k}}{\partial x_{k}}\right) \delta_{i j}$

Here $\mu_{t}$ is the turbulent viscosity which is obtained as: $\mu_{t}=\rho C_{\mu} \frac{k^{2}}{\varepsilon}, \quad C_{\mu}=0.09$

$\mathrm{k}$ and $\varepsilon$ are the turbulent kinetic energy and turbulent dissipation rate which are obtained from the transport equations:

$$
\begin{aligned}
\frac{\partial}{\partial t}(\rho k)+\frac{\partial}{\partial x_{i}}\left(\rho k u_{i}\right)= & \frac{\partial}{\partial x_{j}}\left[\left(\mu+\frac{\mu_{t}}{\sigma_{k}}\right) \frac{\partial k}{\partial x_{j}}\right] \\
& +G_{k}-\rho \varepsilon-Y_{M}
\end{aligned}
$$

$$
\begin{aligned}
\frac{\partial}{\partial t}(\rho \epsilon)+\frac{\partial}{\partial x_{i}}\left(\rho \varepsilon u_{i}\right)= & C_{\varepsilon 1} \frac{\varepsilon}{k} G_{k}-C_{\varepsilon 2} \rho \frac{\varepsilon^{2}}{k} \\
& +\frac{\partial}{\partial x_{j}}\left[\left(\mu+\frac{\mu_{t}}{\sigma_{\epsilon}}\right) \frac{\partial \varepsilon}{\partial x_{j}}\right]
\end{aligned}
$$

In these equations, $G_{k}$ represents the generation of turbulence kinetic energy due to the mean velocity gradients. $Y_{M}$ represents the contribution of the fluctuating dilatation in compressible turbulence to the overall dissipation rate. $C_{\varepsilon 1}, C_{\varepsilon 2}, \sigma_{k}$ and $\sigma_{\epsilon}$ are the model coefficients which are 1.44, 1.92, 1 and 1.3, respectively. The details of theses equations may be found in Wilcox [38]. As noted before, the train model is located near the tunnel entrance. The ambient on both entrances of the tunnel is at atmospheric pressure. In the computational domain, the finite volume method with the SIMPLE algorithm is applied. The time derivative is discretized by first order implicit scheme while the diffusion and convection terms are discretized by second-order upwind scheme.

\section{Results and discussion}

\subsection{Validation of the model}

To check the validity, the results of present numerical model were compared with the experimental data of Ricco et al. [39]. They used a 1/87 scale model of a circular tunnel and train in their experimental study. Figure 2 shows a picture of the model for the tunnel and pressure transducers used in their experimental investigation. They used a 6-m-long tunnel with an inner diameter of $99 \mathrm{~mm}$. The train model was $600 \mathrm{~mm}$ long and the nose angle of $90^{\circ}$, and a diameter of $38 \mathrm{~mm}$. therefore, the blockage ratio was $\beta=0.147$. The train model velocity was $110 \mathrm{~km} / \mathrm{h}$.

Figure 3 shows the time-variation of the pressure at a measuring point located $900 \mathrm{~mm}$ from the tunnel entrance. A comparison between the numerical model predictions with the experimental data of Ricco et al. [39] is observed in this figure. It is shown that the present simulation results are in good agreement with the experimental 


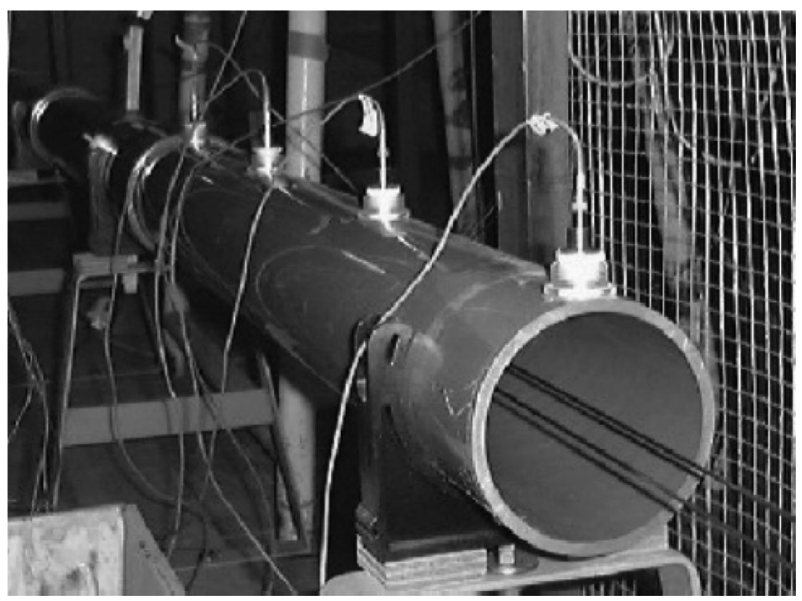

Fig. 2 The tunnel model with pressure transducers used by Ricco et al. [39]

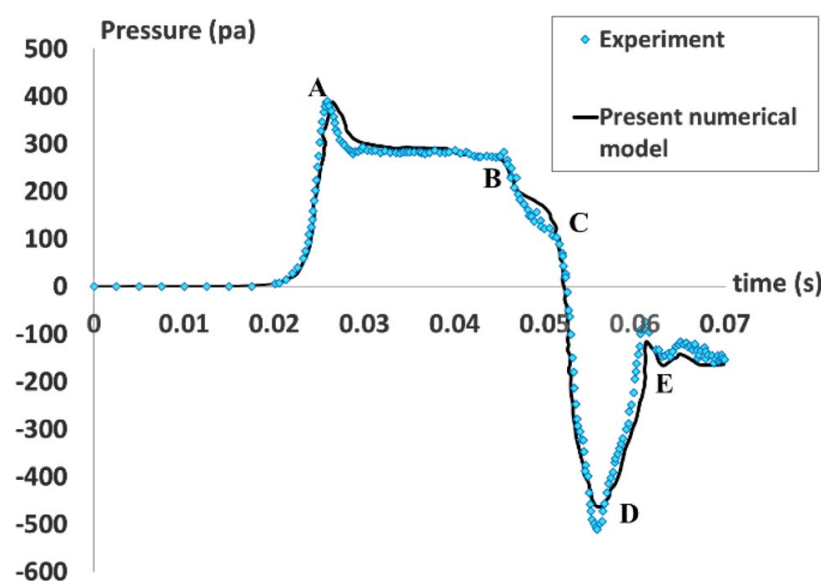

Fig. 3 Comparison between experimental data of Ricco et al. [39] and present numerical results for time variation of pressure at a pressure transducer located $900 \mathrm{~mm}$ from the tunnel entrance

data. When the train head enters the tunnel, a compression wave is generated and propagated along the tunnel. When this compression wave arrives at the location of the pressure transducer, the pressure rises and the first pressure rise is observed $(A)$. The local pressure remains almost constant. But, when the tail of the train enters the tunnel, an expansion wave is generated which decreases the local pressure (B). During the time elapsed by the train in passing through the measuring point, the pressure of the measuring point is decreased (C). On the other hand, the first compression wave arrives at the tunnel outlet, so it is reflected back to the tunnel with the opposite sense (expansion wave). By the arrival of this expansion wave, the minimum value in the pressure of the measuring point is observed (D). Again, the expansion wave arrives at the tunnel entrance and is reflected to the tunnel with the opposite sense, this causes the pressure to be increased (E).

Now that the numerical method is validated, the influence of some parameters, such as flow compressibility, train and tunnel cross-sectional shapes, train acceleration and train length on the maximum pressure rise, are investigated.

\subsection{Compressibility effect}

In the present numerical model, the compressible forms of the governing equations for the flow field are solved. The Mach number based on the train velocity for the studied cases is relatively small. For instance, when the train velocity is $30 \mathrm{~m} / \mathrm{s}$, the Mach number is 0.08 . Despite the low Mach number values, the compressibility effect should be considered due to the importance of pressure wave propagation in the tunnel. A detailed discussion of this issue was presented by Ricco et al. [39]. A brief summary is provided in this section.

The use of a compressible flow model is necessary due to the presence of the two time scales in this flow problem. The first scale is the time the train body needs to get into the tunnel $\left(\mathrm{t}_{1}=\mathrm{L}_{\text {train }} / \mathrm{V}_{\text {train }}\right)$, and the second scale is the time the pressure wave needs to propagate along the tunnel at the sound speed $\left(t_{2}=L_{\text {tunnel }} / a\right)$. In fact, the pressure waves are generated at the tunnel entrance and propagate with the speed of sound. So the time in which the generated pressure waves reach the tunnel exit and reflect back into the tunnel with opposite sense, depends on the length of the tunnel, $L_{\text {tunnel, }}$ and the speed of sound, a. Therefore, the important parameters that affect the wave propagation and airflow distribution are train velocity, train length, tunnel length, and the speed of sound. Ricco et al. [39] stated that if the two noted time scales are of the same order, then the compressibility is important and must be taken into account to study the flow. This statement is evaluated for some cases in the present work.

To emphasize the importance of using a compressible flow model, an incompressible model is used to solve the case studied by Ricco et al. [39]. In this case study, the two time scales are of the same order $\left(t_{1}=t_{2} \sim 0.02 \mathrm{~s}\right)$. Figure 4 Compares the predicted pressure variations by the incompressible model with those of the compressible model. It is shown in this figure that the predictions of the compressible model are in good agreement with experimental results of Ricco et al. [39], while the incompressible model cannot predict the interaction of the expansion wave (which was generated as the reflection of the incoming pressure wave from the tunnel exit) with the flow field in the tunnel $(t \geq 0.05)$. In addition, after the train passes the measuring point, several fluctuations can be seen in the time variation of the pressure. These fluctuations, resulting 
Fig. 4 Comparing the time variation of pressure for both compressible and incompressible models in tunnels with circular cross sections. a The case of experimental work of Ricco et al. [39]. b A case with train length of $100 \mathrm{~m}$, tunnel length of $1000 \mathrm{~m}$, blockage ratio of $\beta=0.338$, train velocity of $V=4.472 \mathrm{~m} / \mathrm{s}$

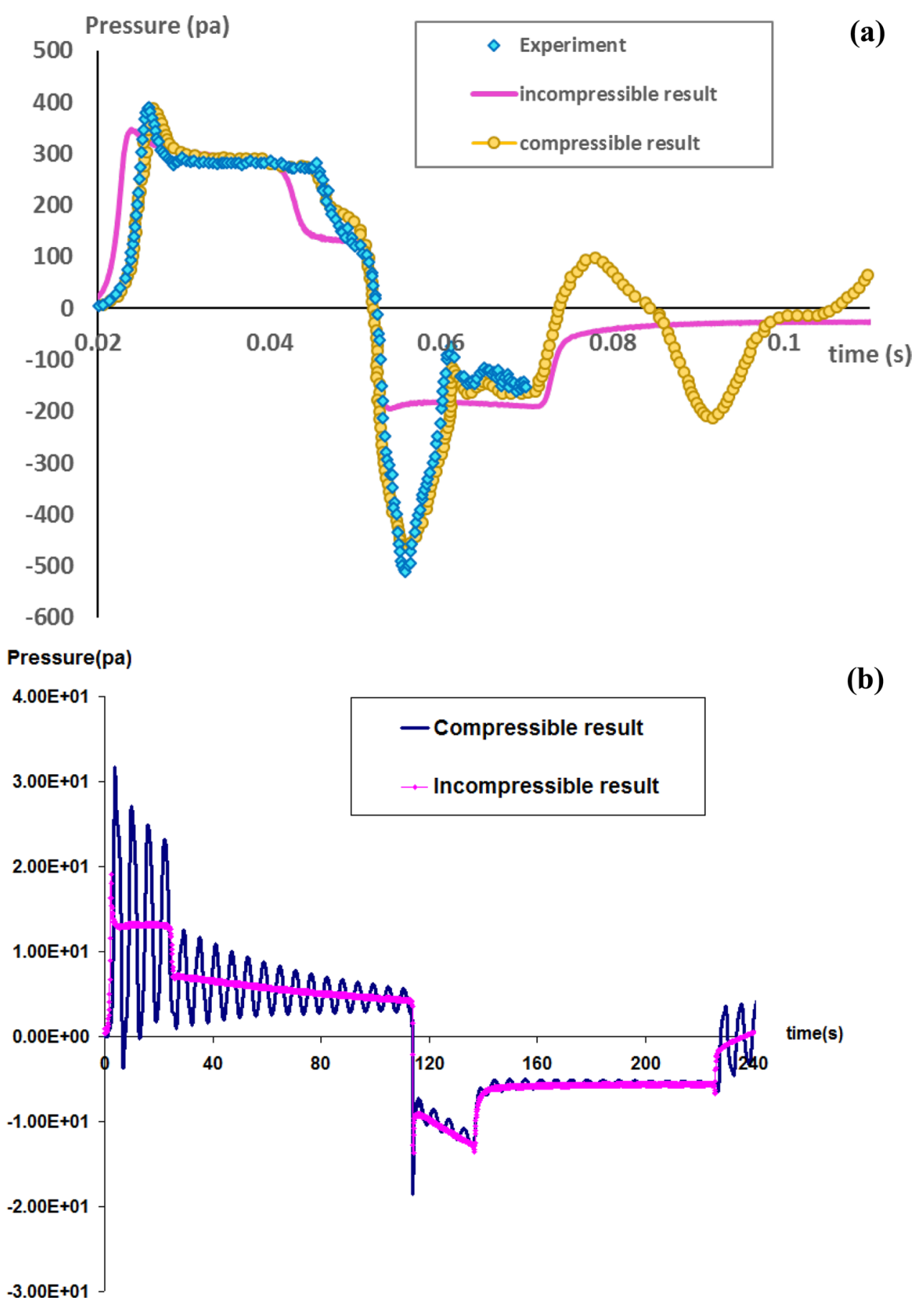

from the effect of waves traveling and reflecting along the tunnel, cannot be predicted properly by an incompressible numerical model.

To show another example for the importance of the compressibility effect, a train model, $100 \mathrm{~m}$ long, is considered which moves along a $1000 \mathrm{~m}$ tunnel. In this case, the blockage ratio is $\beta=0.338$, and the train velocity is $\mathrm{V}=4.472 \mathrm{~m} / \mathrm{s}$. Here, the inlet velocity is low; therefore, the generated pressure waves are weak compared to the previous example. The time variations of pressure obtained for both compressible and incompressible models were compared in Fig. 4b. for the measuring point, located $500 \mathrm{~m}$ from the tunnel entrance. As noted before, compressibility is important when the two time scales, $t_{1}$ and $t_{2}$, are of the same order. For this case, however, the time scales are not of the same order, $t_{1}=22.4 \mathrm{~s}$ and $t_{2}=2.8 \mathrm{~s}$. As Ricco et al. [39] suggested, an incompressible model should be capable of properly predicting the pressure variation. 
When the tunnel length or train velocity is small $\left(t_{1} \gg t_{2}\right)$, the pressure waves travel along the tunnel length and reflect back into the tunnel several times before the train can completely enter the tunnel. This leads to fluctuations in the pressure. The average of pressure fluctuations for the compressible flow case is equal to the pressure magnitude predicted by the incompressible model. Here, the pressure waves generated, due to the train's entering the tunnel, travel and reflect back into the tunnel several times before the train tail enters the tunnel due to low train velocity. When the train tail enters the tunnel at $\mathrm{t} \approx 22.5 \mathrm{~s}$, an expansion wave is generated and propagated, which decreases the pressure. The intensity of the fluctuations decays with time as part of the energy is released to the ambient as a micro-pressure wave. The sudden decrease of the pressure to a minus gauge pressure occurs when the train head passes the measuring point at $t \approx 112 \mathrm{~s}$. The pressure starts to rise again when the train tail reaches the measuring point at $t=135 \mathrm{~s}$. The pressure is constant until the train exits the tunnel. The pressure starts to increase and fluctuate again after the train exits the tunnel. Therefore, even when the two noted time scales are not of the same order, a compressible model is more suitable, because it can predict the pressure fluctuations. An incompressible model, however, can be used for predicting the mean pressure fluctuations. The effect of compressibility, when the train is accelerating is a subject that will be further explained.

\subsection{Train and tunnel shape}

Many investigations believed that the pressure waves generated and propagated in the tunnels due to the entrance and motion of trains are planar, and a 1-D model would be sufficient for evaluating the time variation of pressure at a section of the tunnel. Ricco et al. [39] showed that the flow can be simulated by a one-dimensional code when no recirculation region is present around the train head. Izadi et al. [19] proposed an axisymmetric model which can well predict the results of complicated three dimensional flow inside subway system. For some cases, however, these pressure waves are not planar, and 3-D models are needed to evaluate their effects. Here this point is investigated, and the results of an axisymmetric model are compared with the 3-D model. In fact, with this comparison, the effect of train and tunnel cross-sectional shape on the maximum pressure rise is also investigated.

\subsubsection{Cubic train and tunnel in comparison with axisymmetric train and tunnel}

In this section, the experimental work of Kim and Kim [12], for a tunnel and train with rectangular cross sections, is selected for studying the shape effect. The train starts with an acceleration of $1 \mathrm{~m} / \mathrm{s}^{2}$ and reaches a maximum velocity of $3.0 \mathrm{~m} / \mathrm{s}$. After moving at this speed for $10 \mathrm{~s}$, the train decelerates with an acceleration of $-1 \mathrm{~m} / \mathrm{s}^{2}$. The schematic of their experimental model with the size of train and tunnel model is illustrated in Fig. 5a. The blockage ratio is 0.669 . Then, an axisymmetric model of the train and tunnel, with the same blockage ratio and train speed is simulated. An axisymmetric model which represents the circular train and tunnel model can be compared to the model with rectangular cross sections. The train model diameter is assumed to be $0.186 \mathrm{~mm}$, and the tunnel model diameter is $0.228 \mathrm{~mm}$. Therefore, the blockage ratio will be the same. Also, the length of train and tunnel model for both cubic and axisymmetric models are the same.

The time variations of $C_{p}$ at a point on the ceiling, located $8.5 \mathrm{~m}$ from the tunnel inlet (PT1), are shown in Fig. $5 \mathrm{~b}$. As can be seen from this figure, the resulting pressure distributions for the cubic and axisymmetric models of the tunnels and trains are roughly the same, which implies that for the same blockage ratio, the tunnel and the train cross sectional shapes do not affect the maximum pressure rise or the pressure distributions in the tunnel and the most important geometrical parameter is the blockage ratio.

\subsubsection{Cubic train moving in partially circular tunnel in comparison with circular train and tunnel}

In this section, one of the tunnels in Shiraz subway system, shown schematically in Fig. 1, is considered for analysis. The tunnel length is $1000 \mathrm{~m}$ and two $120 \mathrm{~m}$ stations are present at the sides of the tunnel. The train is placed $10 \mathrm{~m}$ from the tunnel inlet. The tunnel cross section has a diameter of $6 \mathrm{~m}$, and the train model is $100 \mathrm{~m}$ long, $2.7 \mathrm{~m}$ wide, and $3.4 \mathrm{~m}$ high. The blockage ratio is 0.338 and the train speed is $20 \mathrm{~m} / \mathrm{s}$. Then, by applying an axisymmetric model, a train and tunnel model with circular cross sectional shape but with similar blockage ratio and speed is simulated. The train model diameter is $3.48 \mathrm{~m}$ and the tunnel model diameter is $6 \mathrm{~m}$. Besides, for a more accurate comparison, a 2-D model with the same velocity and blockage ratio is also considered. Time variations of pressure at a point located $200 \mathrm{~m}$ from the tunnel inlet are compared for these three models and shown in Fig. 6 . As can be seen in this figure, there are no considerable differences between the results of 3-D and axisymmetric models but the results of 2-D model are not in a good agreement with 3-D model. So the best simple substitution for 3-D model is an axisymmetric model with the same blockage ratio. Therefore, in the following sections, the effects of train acceleration and length are investigated using axisymmetric models. 

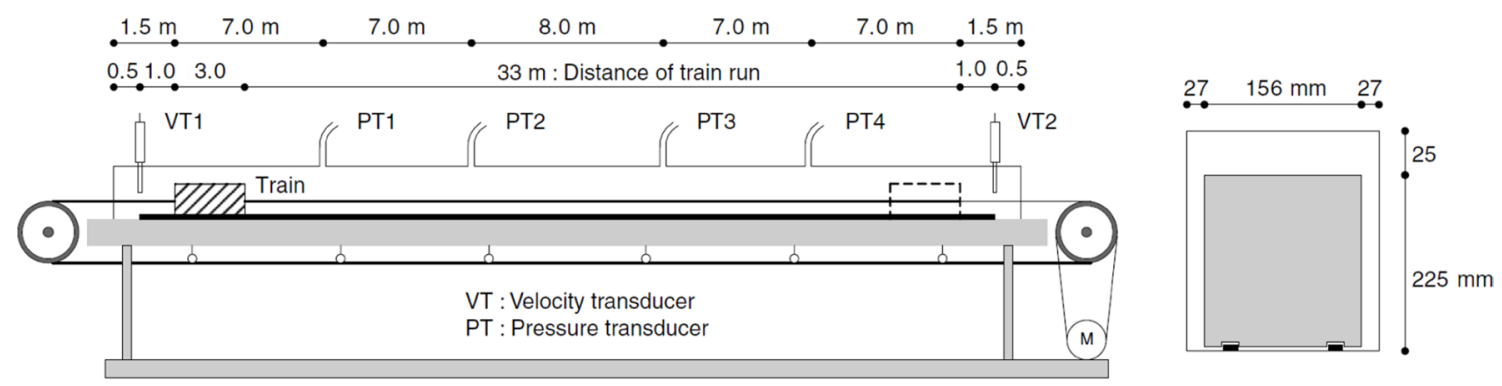

(a)

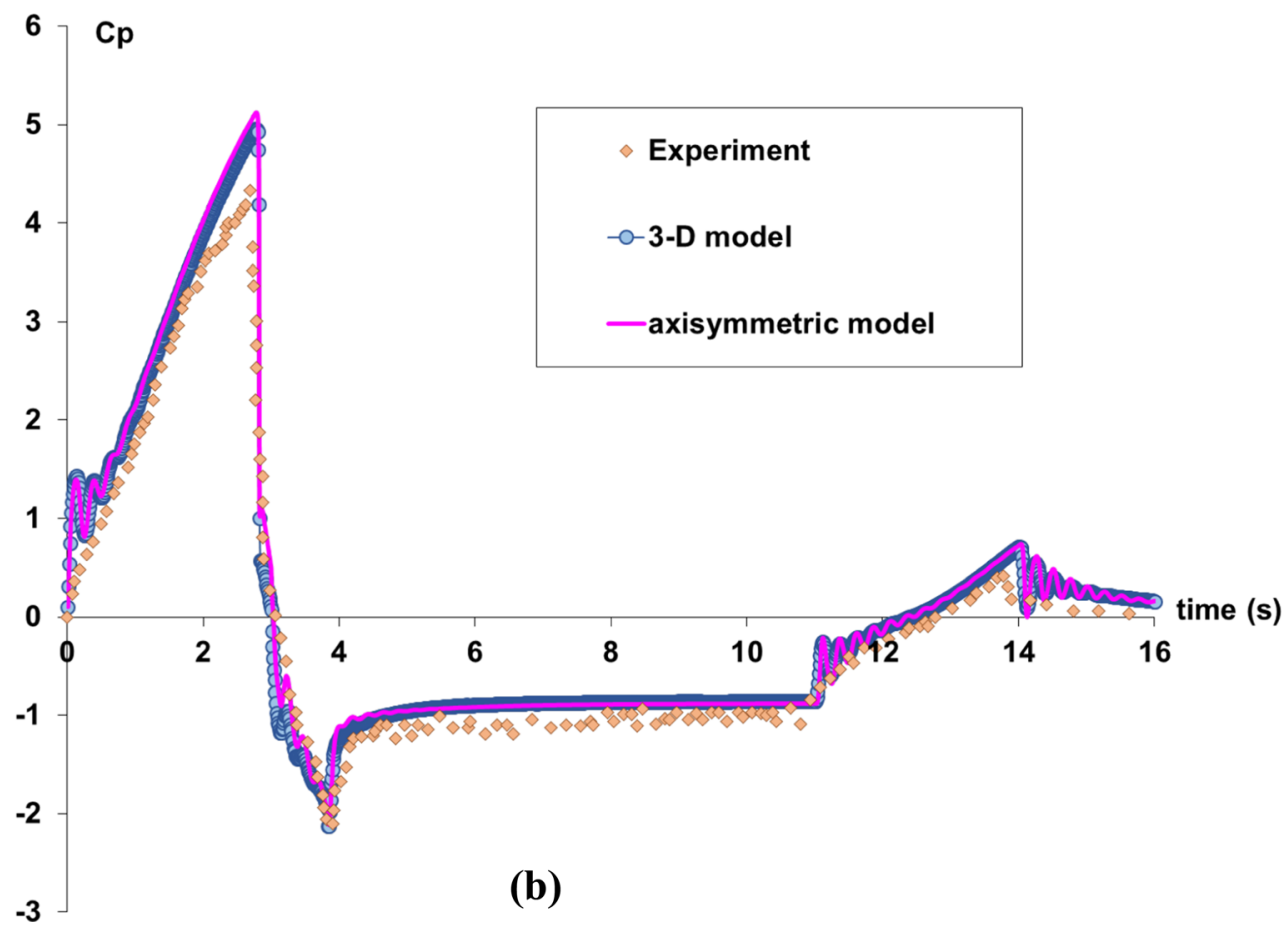

Fig. 5 a Schematic diagram of the experimental model of Kim and Kim [12]. b Comparison of the present numerical model predictions with the experimental data and numerical simulation of Kim and Kim [12] for time variation of Cp at the location of pressure transducer PT1

\subsection{Train acceleration}

One important parameter affecting the first and maximum pressure rise inside the tunnel is the train entering velocity. In most previous studies, the pressure rise is estimated for trains moving with constant velocity. In fact, in all previously presented equations for pressure rise in a tunnel, the constant velocity of the train is an input parameter. However, the influence of train acceleration on the first pressure rise, and the maximum pressure rise in the tunnel has not been reported in the open literature so far.

To study the effect of acceleration, an axisymmetric model was used to analyze the Shiraz subway system with a blockage ratio of 0.338 . The train velocity is shown in Fig. 7a. In these cases, the final velocity of the train is $20 \mathrm{~m} / \mathrm{s}$, while the acceleration of the train is assumed to be $2,1.5,1$ and $0.5 \mathrm{~m} / \mathrm{s}^{2}$, which are realistic magnitudes.
Figure $7 \mathrm{~b}$ illustrates the time-variations of pressure at a specified measuring point, located on the tunnel ceiling, $200 \mathrm{~m}$ from the entrance of the tunnel.

As noted before, a compression wave is generated, as the train head enters the tunnel. When the compression wave arrives at the specified point, the first pressure rise is observed which corresponds to the entering velocity of the train (point A in Fig. 7b). When the train body is getting into the tunnel with acceleration, a higher pressure increase occurs. Figure $7 \mathrm{~b}$ clearly shows the impact of train acceleration on the pressure rise from $A$ to $B$.

Based on the results shown in Fig. 7b, it is concluded that for accelerating trains, the first pressure rise does not depend only on the entering velocity of the train. As noted before, various available equations suggested by Pope and Woods [9], Howe [10] and Matsuo et al. [11] use the train's entering velocity into the tunnel. The 
Fig. 6 Comparison of prediction of 3-D, 2-D and axisymmetric models for time variations of pressure at a point located $200 \mathrm{~m}$ from the tunnel inlet of Shiraz subway system

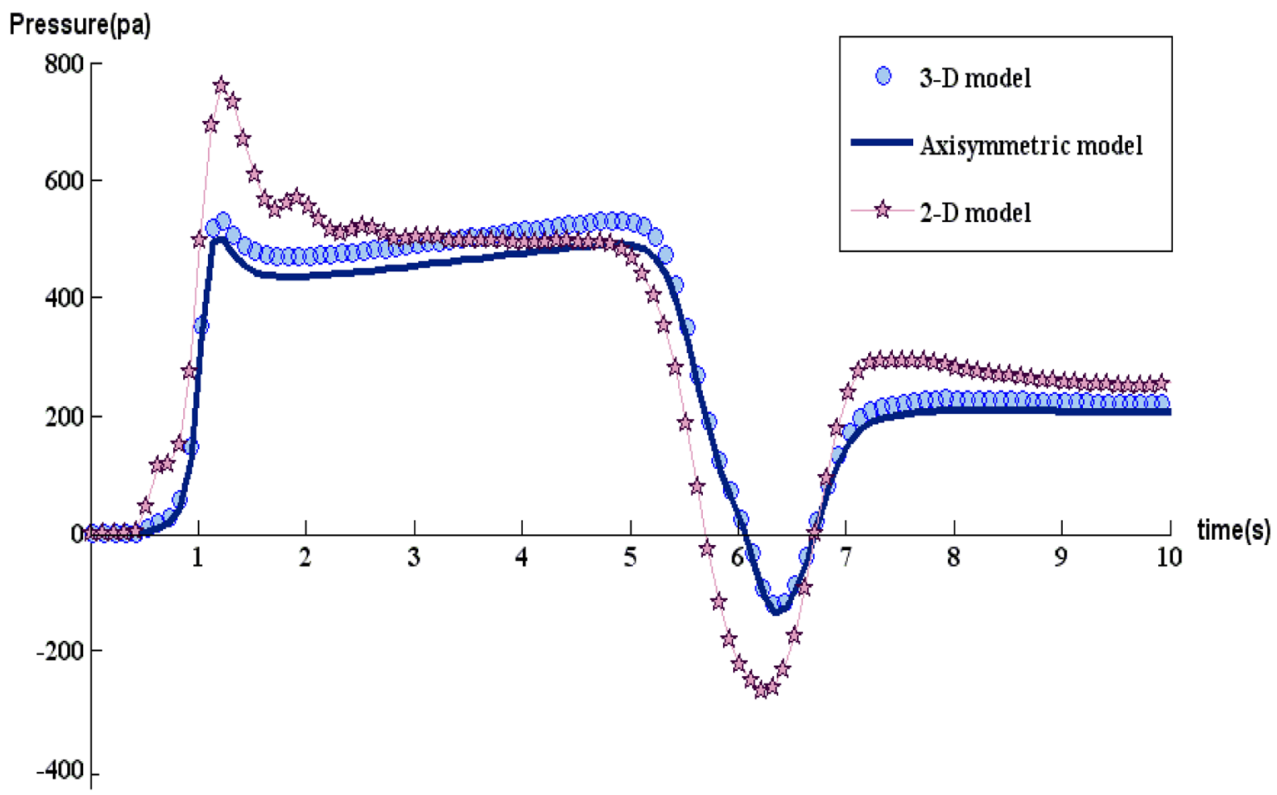

presented results for the accelerating trains, however, show that the use of final velocity $(20 \mathrm{~m} / \mathrm{s})$ is not correct. Because, the train final velocities are the same in all cases, but the time variations of the first pressure rise are quite different. Besides, it can be seen that the initial slope of the pressure curve is proportional to the train acceleration and the increase in train acceleration, increases the first pressure rise. This means that for a train with acceleration of $a=2 \mathrm{~m} / \mathrm{s}^{2}$, the first pressure rise is about $420 \mathrm{~Pa}$ while for a train with acceleration of $\mathrm{a}=1 \mathrm{~m} / \mathrm{s}^{2}$, the first pressure rise is about $250 \mathrm{~Pa}$. Therefore, the first pressure rise depends on the train acceleration.

It should be mentioned that, in some of these cases, the train does not reach exactly its final velocity while entering the tunnel; the train reaches its final velocity of $20 \mathrm{~m} / \mathrm{s}$ when it is entirely in the tunnel. When the train acceleration is $1.5 \mathrm{~m} / \mathrm{s}^{2}$, its tail enters the tunnel with a velocity of $18.166 \mathrm{~m} / \mathrm{s}$. When its acceleration is $1 \mathrm{~m} / \mathrm{s}^{2}$, its tail enters the tunnel with a velocity of $14.83 \mathrm{~m} / \mathrm{s}$. When its acceleration is $0.5 \mathrm{~m} / \mathrm{s}^{2}$, its tail enters the tunnel with a velocity of $10.49 \mathrm{~m} / \mathrm{s}$. But when the train acceleration is $2 \mathrm{~m} / \mathrm{s}^{2}$, its tail enters the tunnel with velocity of $20 \mathrm{~m} / \mathrm{s}$, it means that the train reaches its final velocity while it is entering the tunnel.

In these cases, if the corresponding train tail velocities are used in the equation presented by Pope and Woods [9], $\Delta p=p-p_{0} \approx \frac{\rho U^{2}}{2} \frac{2 \beta}{1-2 \beta}$, the magnitude of the maximum pressure rise is correctly evaluated. Using other velocities, such as the train's entering or final velocity, leads to an incorrect value of the pressure rise in the tunnel. The values of maximum pressure rise predicted by the Pope et al. equation using the train tail velocity are shown by broken lines in Fig. 8a-c and are compared with the results of the present simulation.

Figure $8 a$ shows that for $a=1 \mathrm{~m} / \mathrm{s}^{2}$, the first pressure rise computed by Pope et al. equation is near $250 \mathrm{~Pa}$. In this case the train velocity when its tail enters the tunnel is $14.8 \mathrm{~m} / \mathrm{s}$. When a simulation with a constant velocity of $14.8 \mathrm{~m} / \mathrm{s}$ is performed, the same pressure rise is obtained. Figure $8 \mathrm{~b}$ shows that the first pressure rise for the train with an acceleration of $1.5 \mathrm{~m} / \mathrm{s}^{2}$ is about $360 \mathrm{~Pa}$. This pressure rise is the same as that for a train with a constant velocity of $18.17 \mathrm{~m} / \mathrm{s}$, which is the train tail velocity for the case with an acceleration of $a=1.5 \mathrm{~m} / \mathrm{s}^{2}$. The same result can be seen in Fig. $8 \mathrm{c}$ for a train with acceleration of $a=2 \mathrm{~m} / \mathrm{s}^{2}$. It should be mentioned that in this case $\left(a=2 \mathrm{~m} / \mathrm{s}^{2}\right)$, the train reaches its final velocity of $20 \mathrm{~m} / \mathrm{s}$ while entering the tunnel and so the first pressure rise is the same as that of a train with constant velocity of $20 \mathrm{~m} / \mathrm{s}$. Therefore, for a train acceleration higher than $a=2 \mathrm{~m} / \mathrm{s}^{2}$, the first pressure rise will be the same (about $420 \mathrm{~Pa}$ ), because the train reaches its final velocity while entering the tunnel.

The results further show that, the maximum pressure rise depends on the train velocity when its tail enters the tunnel. This can be justified with the following reasoning: when the train enters the tunnel, it makes a pressure rise according to its entering velocity; but because of train acceleration, the pressure would not remain constant in this range and the pressure continues to rise until the train tail enters the tunnel. The generation of an expansion wave, due to entering the train tail, decreases the pressure. Therefore, the first pressure rise can be computed by the train's tail velocity. 
Fig. 7 a Time variations of train velocity at different accelerations. b Comparing the pressure at a specified point for different train accelerations

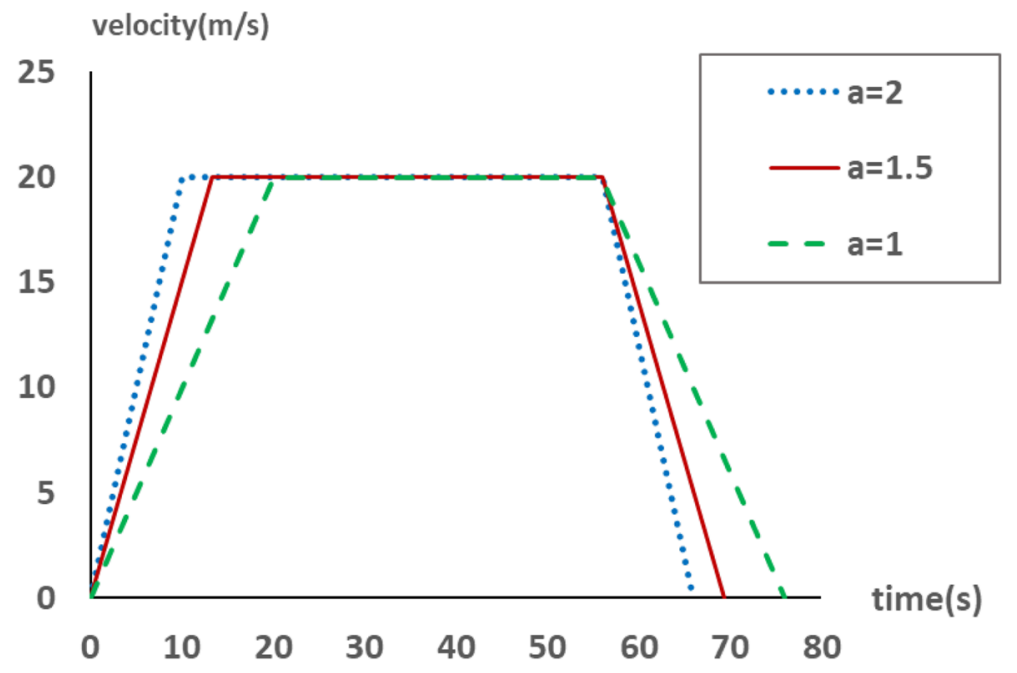

(a)

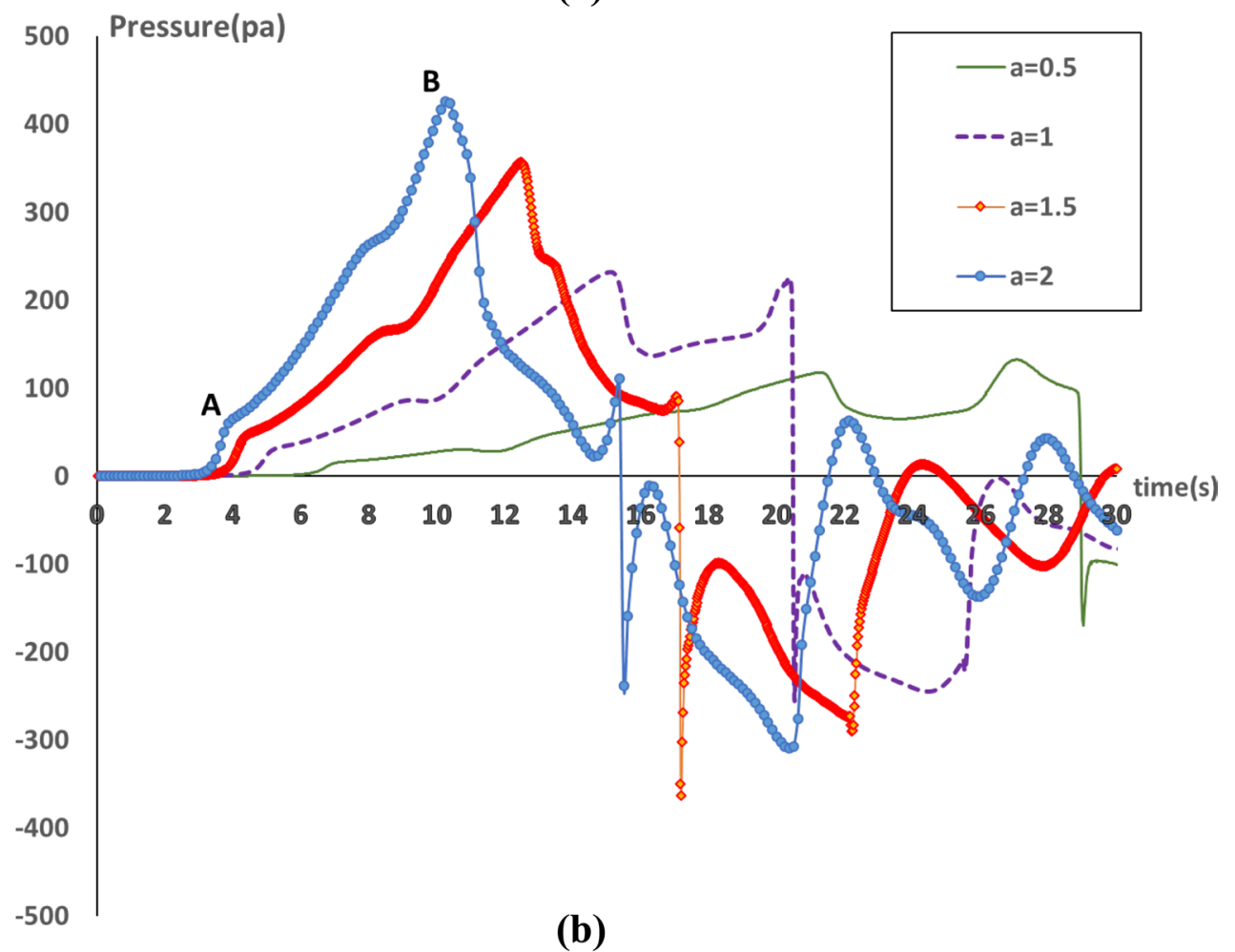

\subsection{Compressibility effect in a flow induced by an accelerating train}

To investigate the effect of compressibility in a flow induced by an accelerating train, the incompressible models are considered and the results of pressure at a specified measuring point are compared in Fig. 9 for two values of acceleration. In incompressible models, the pressure waves are not generated but when the train head with high pressure arrives at the tunnel, it pushes the front air and increases the pressure in the specified measuring point. When the train rear with low pressure enters the tunnel, it causes the pressure of the measuring point to be decreased. By the arrival of train head to the measuring point, the pressure decreases sharply to a negative value. After the train passes by, the flow expands in the wake region and the pressure increases again. These pressure variations can be well predicted using the incompressible model. But the pressure fluctuations cannot be obtained by incompressible model. Because, these fluctuations are created by the pressure 


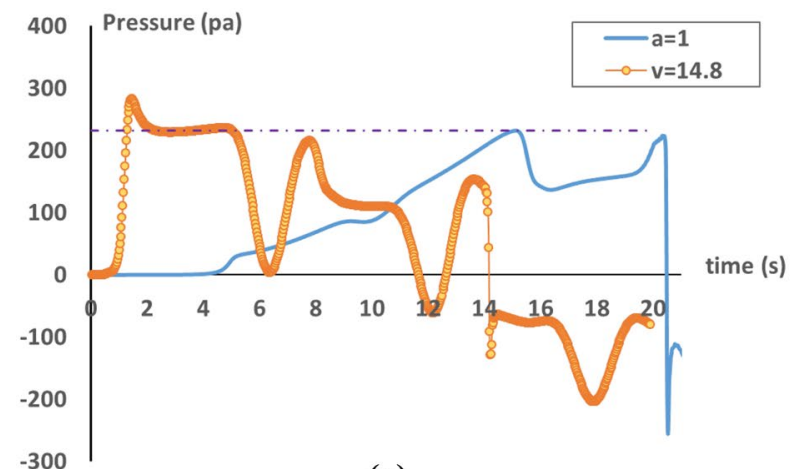

(a)
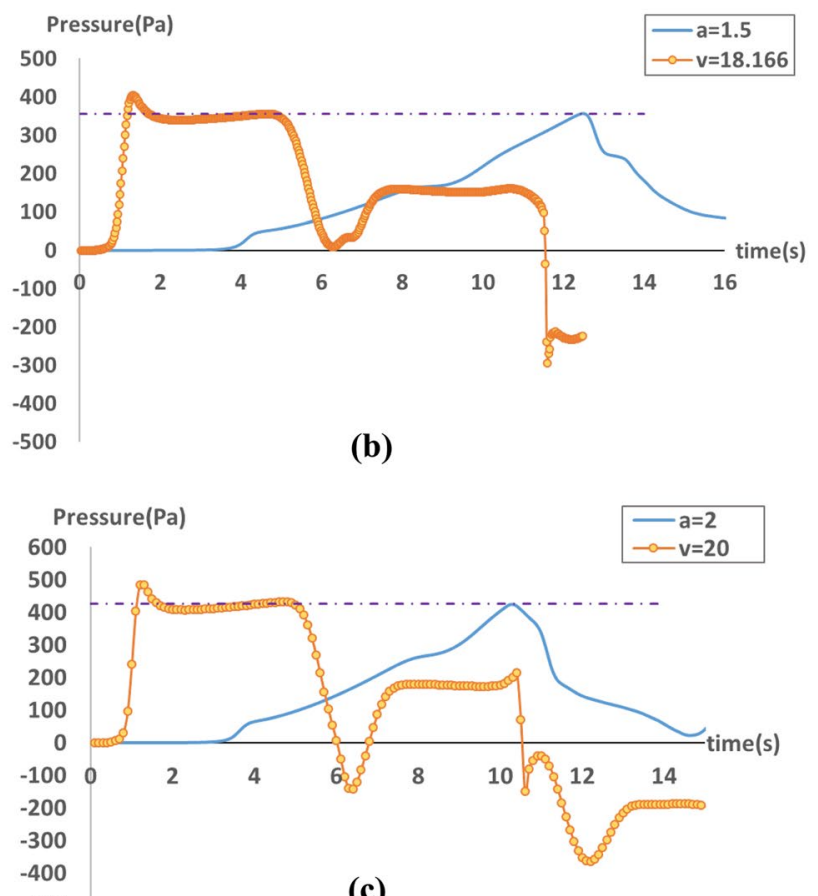

(c)

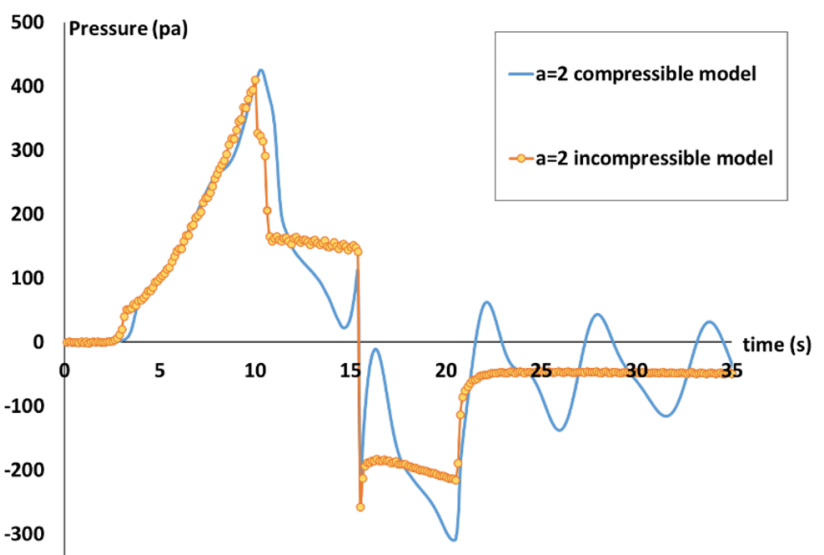

(a)

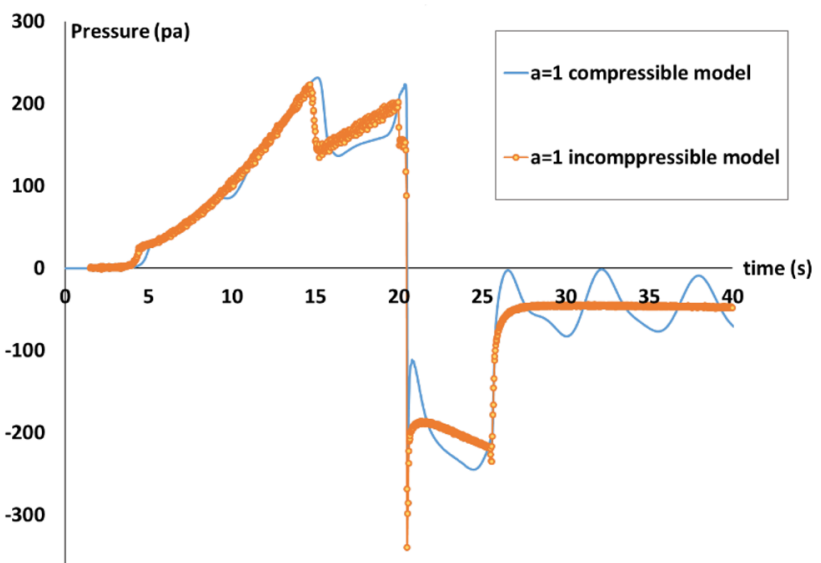

(b)

Fig. 9 Comparison of time variations of pressure for compressible and incompressible models of accelerating train at a specified measuring point $\mathbf{a} a=2 \mathrm{~m} / \mathrm{s}^{2} \cdot \mathbf{b} a=1 \mathrm{~m} / \mathrm{s}^{2}$

\subsection{Effects of train length}

To investigate the effect of train length on the pressure rise in a tunnel, train lengths of $L=50,80$ and $100 \mathrm{~m}$ are simulated using an axisymmetric model of the Shiraz subway system, with a tunnel length of $1000 \mathrm{~m}$ and a blockage ratio of 0.338 . The results show that the train length does not noticeably influence the first pressure rise. Although the time evolutions of pressure are different, the maximum pressure rise is roughly the same. But for the cases with the same acceleration and different lengths, the first pressure rise differs due to changes in train velocity when its tail enters the tunnel. In fact, the time of generation and propagation of an expansion wave is different. The results are not shown here for the sake of brevity. 


\section{Conclusions}

Using the dynamic mesh technique, train movements along tunnel were simulated, and the characteristics of the piston effect inside tunnel were investigated.

The numerical results were compared with experimental data and the results showed that the compressible case model presents more accurate results than the incompressible one.

Comparing the 2-D, axisymmetric, and 3-D model predictions with the same blockage ratio, confirmed that an axisymmetric model can predict the pressure rise of the 3-D model.

The pressure rise due to the motion of accelerating trains was evaluated. The simulation results revealed that the first pressure rise inside the tunnel depends on the train's tail velocity. This finding is in contrast to the belief that the train's nose velocity entering the tunnel is the key parameter for controlling the pressure rise. Therefore, the train tail velocity must be used in the pressure rise equations. From the point of view of compressibility effect in a flow induced by an accelerating train, it was shown that the amplitude of pressure fluctuations is almost small. Thus, the incompressible model can be used especially for a train with low acceleration.

\section{Compliance with ethical standards}

Conflict of interest The authors declare that they have no conflict of interest.

\section{References}

1. Ogawa T, Fujii K (1997) Numerical investigation of threedimensional compressible flows induced by a train moving into a tunnel. Comput Fluids 26:565-585

2. Howe M, Cox E (2005) Reflection and transmission of a compression wave at a tunnel portal. J Fluids Struct 20:1043-1056

3. Baron A, Molteni P, Vigevano L (2006) High-speed trains: prediction of micro-pressure wave radiation from tunnel portals. J Sound Vib 296:59-72

4. Vardy AE (2008) Generation and alleviation of sonic booms from rail tunnels. Proc Inst Civ Eng Eng Comput Mech 161:107-119

5. Nakao S, Aoki T, Yasunobu T, Matsuo K (2001) A study on transition from a compression wave to a shock wave in a high-speed train tunnel. Theoret Appl Mech 50:359-364

6. Takayama K, Sasoh A, Onodera O, Kaneko R, Matsui Y (1995) Experimental investigation on tunnel sonic boom. Shock Waves 5:127-138

7. Iida M, Kikuchi K, Fukuda T (2006) Analysis and experiment of compression wave generated by train entering tunnel entrance hood. JSME Int J Ser B 49:761-770
8. Chen T, Lee Y, Hsu C-C (1998) Investigations of piston-effect and jet fan-effect in model vehicle tunnels. J Wind Eng Ind Aerodyn 73:99-110

9. C. Pope, W. Woods, Boundary conditions for the transit of a train through a tunnel with special reference to the entry and exit mesh fraction and the contact surface, Aerodynamics and Ventilation of Vehicles Tunnel (1992) 79-105

10. Howe M (1998) The compression wave produced by a highspeed train entering a tunnel. Proc R Soc Lond Ser A Math Phys Eng Sci 454:1523-1534

11. Mashimo S, Nakatsu E, Aoki T, Matsuo K (1995) Entry compression wave generated by a high-speed train entering a tunnel. Trans Jpn Soc Mech Eng Part B 61:3720-3727

12. Kim J, Kim K (2007) Experimental and numerical analyses of train-induced unsteady tunnel flow in subway. Tunn Undergr Space Technol 22:166-172

13. Yang W, Deng E, Lei M, Zhang P, Yin R (2018) Flow structure and aerodynamic behavior evolution during train entering tunnel with entrance in crosswind. J Wind Eng Ind Aerodyn 175:229-243

14. Khayrullina A, Blocken B, Janssen W, Straathof J (2015) CFD simulation of train aerodynamics: train-induced wind conditions at an underground railroad passenger platform. J Wind Eng Ind Aerodyn 139:100-110

15. Chen Z, Liu T, Zhou X, Niu J (2017) Impact of ambient wind on aerodynamic performance when two trains intersect inside a tunnel. J Wind Eng Ind Aerodyn 169:139-155

16. Huang Y-D, Wei G, Chang-Nyung K (2010) A numerical study of the train-induced unsteady airflow in a subway tunnel with natural ventilation ducts using the dynamic layering method. $J$ Hydrodyn Ser B 22:164-172

17. Liu M, Zhu C, Cui T, Zhang H, Zheng W, You S (2018) An alternative algorithm of tunnel piston effect by replacing threedimensional model with two-dimensional model. Build Environ 128:55-67

18. Zhang H, Zhu C, Liu M, Zheng W, You S, Li B, Xue P (2017) Mathematical modeling and sensitive analysis of the train-induced unsteady airflow in subway tunnel. J Wind Eng Ind Aerodyn 171:67-78

19. Izadi T, Mehrabian MA, Abouali O, Ahmadi G (2019) 3-D numerical analysis of train-induced flow inside four ventilated underground subway stations and connecting tunnels. JWind Eng Ind Aerodyn 193:103974

20. Bazdidi-Tehrani F, Abouata A, Hatami M, Bohlooli N (2016) Investigation of effects of compressibility, geometric and flow parameters on the simulation of a synthetic jet behaviour. Aeronaut J 120:521-546

21. Chu C-R, Chien S-Y, Wang C-Y, Wu T-R (2014) Numerical simulation of two trains intersecting in a tunnel. Tunn Undergr Space Technol 42:161-174

22. Xue P, You S, Chao J, Ye T (2014) Numerical investigation of unsteady airflow in subway influenced by piston effect based on dynamic mesh. Tunn Undergr Space Technol 40:174-181

23. Camelli FE, Byrne G, Löhner R (2014) Modeling subway air flow using CFD. Tunn Undergr Space Technol 43:20-31

24. Wei-chao Y, Li-min P, Li-chuan W (2009) Computation simulation on aerodynamic characteristic of PSD in subway platform. In: 2009 International conference on computer engineering and technology

25. Jian-jun $L$ (2016) Study on the aerodynamic characteristics of tunnel and metro station with PSD during high-speed train passing tunnel. Int J Signal Process Image Process Pattern Recognit 9:379-392

26. Zhang X, Ma J, Li A, Lv W, Zhang W, Li D (2019) Ventilation for subway stations with adjustable platform doors created by train-induced unsteady airflow. Build Environ 152:87-104 
27. Zhou Y, Yang Y, Mao Z, Bu R, Gong J, Wang Y, Yi L (2019) Analytical and numerical study on natural ventilation performance in single-and gable-slope city tunnels. Sustain Cities Soc 45:258-270

28. Niu J, Zhou D, Liang X, Liu T, Liu S (2017) Numerical study on the aerodynamic pressure of a metro train running between two adjacent platforms. Tunn Undergr Space Technol 65:187-199

29. Niu J-Q, Zhou D, Liang X-F, Liu S, Liu T-H (2018) Numerical simulation of the Reynolds number effect on the aerodynamic pressure in tunnels. J Wind Eng Ind Aerodyn 173:187-198

30. Niu J, Wang Y, Zhang L, Yuan Y (2018) Numerical analysis of aerodynamic characteristics of high-speed train with different train nose lengths. Int J Heat Mass Transf 127:188-199

31. Kim J-Y, Kim K-Y (2009) Effects of vent shaft location on the ventilation performance in a subway tunnel. J Wind Eng Ind Aerodyn 97:174-179

32. Huang Y-D, Chan L, Chang NK (2012) A numerical analysis of the ventilation performance for different ventilation strategies in a subway tunnel. J Hydrodyn Ser B 24:193-201

33. Wang F, Yin Z, He K (2009) A study on subway tunnel ventilation for piston effect. J Am Soc Civ Eng. In: ICPTT, pp 910-921

34. Cross $D$, Hughes $B$, Ingham D, Ma L (2015) A validated numerical investigation of the effects of high blockage ratio and train and tunnel length upon underground railway aerodynamics. J Wind Eng Ind Aerodyn 146:195-206
35. Uystepruyst $D$, William-Louis $M$, Creusé $E$, Nicaise $S$, Monnoyer $F$ (2011) Efficient 3D numerical prediction of the pressure wave generated by high-speed trains entering tunnels. Comput Fluids 47:165-177

36. Rabani M, Faghih AK (2015) Numerical analysis of airflow around a passenger train entering the tunnel. Tunn Undergr Space Technol 45:203-213

37. Wang T, Wu F, Yang M, Ji P, Qian B (2018) Reduction of pressure transients of high-speed train passing through a tunnel by cross-section increase. J Wind Eng Ind Aerodyn 183:235-242

38. Wilcox DC (2006) Turbulence modeling for CFD. DCW Industries Inc, La Canada

39. Ricco P, Baron A, Molteni P (2007) Nature of pressure waves induced by a high-speed train travelling through a tunnel. $J$ Wind Eng Ind Aerodyn 95:781-808

Publisher's Note Springer Nature remains neutral with regard to jurisdictional claims in published maps and institutional affiliations. 